\title{
Extracting Vibration Severity Time Histories from Epicyclic Gearboxes
}

\author{
Juhani Nissilä Esko Juuso \\ Control Engineering, Faculty of Technology, University of Oulu, Finland, P.O.Box 4300, FI-90014 \\ \{juhani.nissila, esko.juuso\} doulu.fi
}

\begin{abstract}
Monitoring epicyclic gearboxes in vital power transition situations is still a challenge. In this paper, we discuss these challenges with long time vibration measurements through two industrial examples. The first are the two gearboxes in the front axle of a load haul dumper (LHD) from Pyhäsalmi mine and the second a two stage gearbox from Kelukoski water power station (WPS). The LHD was monitored almost continuously for nearly two years until its breakdown. The data from WPS was intermittent from a five month period. We discuss how to find stable conditions for comparable measurements in these cases. For this we utilise a tacho signal from the cardan axle of the LHD and power measurements from the WPS. It is found that in both cases second derivatives of acceleration signals, called snap, respond more quickly to changes in vibration severity. In the LHD case we get clear trends for increasing norms of snap signals. The trends are extracted with nonparametric regression. The shorter measurement period of the WPS makes it impossible to say if its changes are only seasonal. Spectral analysis shows increase in high frequency vibration with time in both cases but provides almost no help for detailed diagnostics.
\end{abstract}

Keywords: epicyclic gearbox, spectral analysis, higher derivatives, MIT-indices, nonparametric regression

\section{Introduction}

Typical methods for vibration severity calculations are for example root mean square (rms) values of displacement, velocity or acceleration signals. These may reveal some faults in rotating machines, such as imbalance, but gear and rolling bearing faults often cause high frequency vibration which is more evident in higher derivatives of acceleration. The compact and complex structure of epicyclic gearboxes are no exception. We will present methods to extract vibration severity time histories from epicyclic gearboxes and discuss the difficulties that are encountered.

Signal processing methods are presented in Section 2. These are the Discrete Fourier Transform (DFT) and its inverse (IDFT) for spectral analysis, calculation of derivatives using these transforms, $l_{p}$-norms and MIT-indices for vibration severity calculations and Nadaraya-Watson nonparametric regression for estimation of MIT-trends and other relationships of two variables.

Vibration measurements from the LHD and WPS are described in Section 3 and we will also solve the total revolution times of the gearboxes with the help of some basic number theory. Calculations from vibration measurements are presented in Sections 4 and 5 respectively. Finally the obtained results are discussed in Section 6.

The method for obtaining vibration severity time histories presented in this study is as follows:

1. Find stable and comparable operating conditions of the machine or normalise these conditions computationally.

2. Find which values of norms and derivatives have changed the most during the measurement period and use these for MIT-indices.

3. Use Nadaraya-Watson regression to fill in the gaps in measurements. It also extracts the trends more clearly, since there typically still is some variance left in the calculations. Here the scaling parameter is chosen visually.

\section{Signal processing}

\subsection{DFT and derivatives}

The vibration measurements are stored as sampled sequences $\mathbf{x}=\left(x_{0}, \ldots, x_{N-1}\right)$ of length $T=\Delta t \cdot N$, where $\Delta t$ is the sampling interval. The spectrum of this sampled signal is calculated with the Discrete Fourier transform (DFT)

$$
\mathscr{F}\{\mathbf{x}\}_{k}=X_{k}=\frac{1}{N} \sum_{n=0}^{N-1} x_{n} e^{-i 2 \pi k n / N} .
$$

Its inverse transform (IDFT) is

$$
\mathscr{F}^{-1}\{\mathbf{X}\}_{n}=x_{n}=\sum_{k=0}^{N-1} X_{k} e^{i 2 \pi k n / N} .
$$

Here we have equated the inverse as $x_{n}$, because it returns the original signal at the sample points (Briggs and Henson, 1995). The DFT and IDFT pair can be used for differentiation and integration of signals. An algorithm for this consists of calculating the DFT coefficients $X_{k}$ and 
then forming a new sequence $\mathbf{G}=\left(G_{0}, \ldots, G_{N-1}\right)$, with $G_{0}=0$ and

$$
\begin{aligned}
G_{k} & =\left(\frac{2 \pi k i}{T}\right)^{z} X_{k}, \quad 0<k<N / 2 \\
G_{N+k} & =\left(\frac{2 \pi k i}{T}\right)^{z} X_{N+k}, \quad-N / 2<k<0 \\
G_{N / 2} & =\left(\frac{\pi N}{T}\right)^{z} \cos \left(z \frac{\pi}{2}\right) X_{N / 2} \quad \text { (if } N \text { is even), }
\end{aligned}
$$

where $z$ is the order of derivative (or integral when negative). Finally we get the vector $\mathbf{x}^{(z)}$ with the IDFT

$$
x_{n}^{(z)}=\mathscr{F}^{-1}\{\mathbf{G}\}_{n} .
$$

The only problematic part in deriving this algorithm is the term $G_{N / 2}$ for even $N$, which the author has presented in (Nissilä et al, 2014). The algorithm also works with any complex $z$, in which case we use the principal values of $\left(\frac{2 \pi k i}{T}\right)^{z}$ and $\left(\frac{\pi N}{T}\right)^{z}$. It then calculates an approximation of the Fourier or Weyl fractional derivatives and integrals. This operation is also sometimes called differintegration.

The DFT assumes the sequence periodic and thus it is practical to window the signal by multiplying it with a suitable window function to attenuate any discontinuities at the end points of the sequence. We use the window function which was introduced in (Lahdelma and Kotila, 2005)

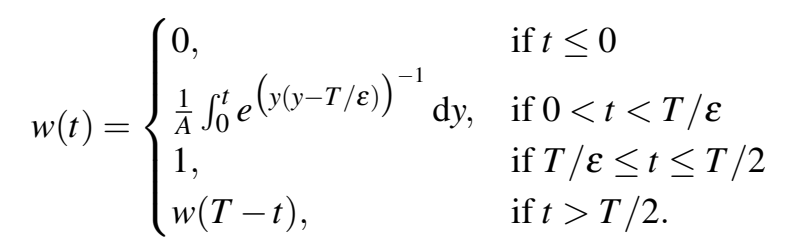

Here $A=\int_{0}^{T / \varepsilon} e^{(y(y-T / \varepsilon))^{-1}} \mathrm{~d} y$ and $\varepsilon$ is the portion of $T$ for ascent and descent. Window function $w$ is infinitely differentiable and, therefore, it preserves the continuity properties of the original signal. We use the trapezoidal rule to approximate the integrals in the definition of $w$.

\section{$2.2 l_{p}$-norms and MIT-indices}

The generalised $l_{p}$-norm or Hölder mean of vector $\mathbf{x}^{(z)}$ is

$$
\left\|\mathbf{x}^{(z)}\right\|_{p, \frac{1}{N}}=\left(\frac{1}{N} \sum_{n=0}^{N-1}\left|x_{n}^{(z)}\right|^{p}\right)^{1 / p}
$$

for $p \geq 1$. This is the traditional $l_{p}$-norm with equal weights $1 / N$. The generalisation to cases $-\infty \leq p \leq \infty$ is done in (Bullen, 2003) with the limiting values

$$
\left\|\mathbf{x}^{(z)}\right\|_{p, \frac{1}{N}}= \begin{cases}\left(\sum_{n=0}^{N-1} \frac{1}{N}\left|x_{n}^{(z)}\right|^{p}\right)^{1 / p} & \text { if } p \in \mathbb{R} \backslash\{0\} \\ \left(\prod_{n=0}^{N-1}\left|x_{n}^{(z)}\right|\right)^{1 / N} & \text { if } p=0 \\ \max _{n=0, \ldots, N-1}\left|x_{n}^{(z)}\right| & \text { if } p=\infty \\ \min _{n=0, \ldots, N-1}\left|x_{n}^{(z)}\right| & \text { if } p=-\infty .\end{cases}
$$

The Hölder mean includes many traditional features, such as minimum value $p=-\infty$, harmonic mean $p=-1$, geometric mean $p=0$, arithmetic mean $p=1$, root mean square (rms) $p=2$ and maximum value $p=\infty$.

The dimensionless MIT-index for vibration severity evaluation was first presented in (Lahdelma, 1992) and it utilised the rms values of integer order derivatives and integrals. It has been generalised to real order differintegrals (Lahdelma and Juuso, 2011) as

$$
{ }^{\tau} M I T_{\alpha_{1}, \alpha_{2}, \ldots, \alpha_{M}}^{p_{1}, p_{2}, \ldots, p_{M}}=\frac{1}{M} \sum_{m=1}^{M} b_{\alpha_{m}} \frac{\left\|\mathbf{x}^{\left(\alpha_{m}\right)}\right\|_{p_{m}, \frac{1}{N}}}{\left\|\mathbf{x}_{\mathrm{ref}}^{\left(\alpha_{m}\right)}\right\|_{p_{m}, \frac{1}{N}}}
$$

where $\alpha_{m}, p_{m} \in \mathbb{R}, \sum_{m=1}^{M} b_{\alpha_{m}}=1, \tau$ is the length of the signal and $\mathbf{x}_{\mathrm{ref}}$ is a reference signal from the machine in good condition or low stress. Typically MIT increases together with decreasing machine condition. The MIT index can also compare stress levels of different operating conditions.

\subsection{Nadaraya-Watson nonparametric regres- sion}

Nonparametric regression of two variables using a kernel function was proposed in 1964 independently by Nadaraya and Watson (Nadaraya, 1964; Watson, 1964). Suppose that we have measured values $\boldsymbol{x}$ at the points $\boldsymbol{t}$. Then the estimated value $x$ at $t$ is

$$
x(t)=\frac{\sum_{n=0}^{N-1} K_{h}\left(t-t_{n}\right) x_{n}}{\sum_{n=0}^{N-1} K_{h}\left(t-t_{n}\right)},
$$

where $K_{h}$ is some non-negative and even kernel function for which $\int_{-\infty}^{\infty} K(t) \mathrm{d} t=1$ and $h$ is a scaling parameter so that the scaled kernel is

$$
K_{h}(t)=\frac{1}{h} K\left(\frac{t}{h}\right) .
$$

Smaller $h$ gives an estimate which follows individual measurements more closely whereas bigger $h$ gives a smoother and more slowly changing function. The kernel in all the regression calculations in this study is a Gaussian (normal distribution)

$$
K(t)=\frac{1}{\sqrt{2 \pi}} e^{-\frac{t^{2}}{2}} .
$$

\section{Measurements and gearbox proper- ties}

\subsection{Load haul dumper front axle}

The measurement setup consisted of four SKF CMPT 2310 accelerometers which were mounted externally onto the LHD's front axle housing and a tachometer on the drive shaft. Accelerometers were located near the planetary gearboxes on either side of the axle and were positioned horizontally and vertically. Measurements were 
recorded with a National Instruments CompactRIO 9024 data logger into a solid-state drive (SSD) as binary files of one minute length. Sampling frequency is $12800 \mathrm{~Hz}$, and a built-in antialising filter guarantees that there are no aliases at frequencies that are less than $0.45 \cdot 12800 \mathrm{~Hz}$ $=5760 \mathrm{~Hz}$. More information on the measurements can be found from (Laukka et al, 2016).

The measurement points are called right vertical (RV), left vertical (LV), right horizontal (RH) and left horizontal $(\mathrm{LH})$. During the first month of the measurements, the accelerometer cables of LV and LH broke down and were replaced. Two SSDs also broke down almost simultaneously after six months of service, which stopped the whole measurement for a month and a half. A third accelerometer at RH broke down during this stoppage and was replaced. Finally six months before the end of measurements, the accelerometer at RH was broken and after that also the accelerometer at RV. There was only one spare accelerometer left which was installed to LV and the accelerometer at RH was moved to RV (since at this point it was clear that the vertical measurements were more sensitive to the deteriorating condition of the axle.)

At the beginning, measurements were always recorded when the LHD was operating. After the stoppage caused by the broken SSDs the program was modified to record only two hours of data after the LHD starts up.

The cardan axle transfers the power first to a differential in the front axle which has a driving pinion with 9 teeth and a crown wheel with 46 teeth (spiral bewel gears). Based only on some pictures, the actual differential operation is carried out with straight bevel gears that probably have 20 teeth. These do not affect the output ratio if the LHD is not turning. Shafts in the front axle then rotate the sun gears in the epicyclic gearboxes on either side. The epicyclic gearboxes consist of a stationary ring gear (104 teeth), three planetary gears (39 teeth) and a sun gear (19 teeth). These are simple spur gears. Unfortunately, we have not yet received enough detailed information about the bearings in the system other than that they are of tapered roller type. The planet carrier provides the output to the front wheel. This is an example of an epicyclic gearbox in the planetary configuration and driven in reduction mode. Assuming then that the rotational frequency of the drive shaft is $v_{\text {driveDIF }}=13.5 \mathrm{~Hz}$, we get the differential gear mesh frequency

$$
v_{\text {meshDIF }}=9 \cdot v_{\text {driveDIF }}=121,5 \mathrm{~Hz} .
$$

If the LHD is not turning, the differential provides the same rotational frequencies to both sides of the axle and the frequencies of the epicyclic gears are (Vicuña, 2010; Immonen et al, 2012) (negative sign means opposite direction)

$$
v_{\text {sunLHD }}=\frac{9}{46} v_{\text {driveDIF }} \approx 2,64 \mathrm{~Hz},
$$

$$
\begin{gathered}
v_{\text {carrierLHD }}=\frac{19}{19+104} v_{\text {sunLHD }}=\frac{19}{123} v_{\text {sunLHD }} \\
=0.408 \mathrm{~Hz}, \\
v_{\text {planet } L H D}=-\frac{104-39}{39} v_{\text {carrier } L H D}=-\frac{5}{3} v_{\text {carrier } L H D} \\
=-\frac{95}{369} v_{\text {sunLHD }} \approx-0.680 \mathrm{~Hz},
\end{gathered}
$$

and the planetary gear mesh frequency

$$
v_{\text {meshLHD }}=104 \cdot v_{\text {carrierLHD }} \approx 42.43 \mathrm{~Hz} \text {. }
$$

Gear tooth numbers are typically relative primes, i.e. their greatest common divisor is 1 . This means that it takes a long time for a gearbox to mesh through all of its tooth pairs. To calculate this time for a full revolution of meshes, we seek whole number solutions for the number of revolutions $N_{\text {sunLHD }}, N_{\text {carrierLHD }}$ and $N_{\text {planetsLHD }}$. This leads to congruence equations

$$
\begin{aligned}
& 19 \cdot N_{\text {sunLHD }}=0 \bmod 123, \\
& 95 \cdot N_{\text {sun } L H D}=0 \bmod 369 .
\end{aligned}
$$

The solution method can be found in any basic number theory book (Strayer, 1994), and they can also be solved with symbolic mathematical software (such as Mathematica) and even solvers for web browsers exist. The solutions of $N_{\text {SunLHD }}$ are $123 \mathrm{~m}$ and $369 \mathrm{~m}$ respectively for all $m \in \mathbb{Z}$. The smallest combined solution is the least common multiple of 123 and 369, which is $\operatorname{lcm}(123,369)=$ 369. Thus after 369 revolutions of the sun gear, every tooth has returned to their original position and this takes $369 \cdot 1 / v_{\text {sunLHD }} \approx 139.70$ seconds. For most situations this means far too long a signal to analyse (if we can even find that long signals with relatively constant speed). So in practise, we analyse signals whose lengths are at least the revolution times of all the individual components.

\subsection{Water power station gearboxes}

The water power station at Kelukoski has a two stage epicyclic gearbox. The first (slower) is called gearbox 1 and the second (faster) will be called gearbox 2. Both were monitored with one WBS CM301 sensor (acceleration data was recorded) with sampling frequency 5000 Hz. Every 15 minutes a signal of length $7 \mathrm{~s}$ was recorded from both measurement points as WAV files. There were four continuous periods of data collection.

Since the WPS is connected to the Finnish power grid, its output frequency is kept at $12,5 \mathrm{~Hz}$ to a high precision (the frequency of the power grid is four times this, i.e. $50 \mathrm{~Hz}$ ) Thus we can calculate the characteristic frequencies backwards starting from the output of the faster gearbox. Gearbox 2 is in the star configuration, meaning that it has a stationary planet carrier with six planet gears (25 teeth). Output is provided via the sun gear (36 teeth), $v_{\text {sunWPS2 }}=12,5 \mathrm{~Hz}$, and input from the gearbox 1 via the 
ring gear (86 teeth). The gear teeth are double helical and the gearboxes have plain bearings. The formulas for the frequencies of these components are particularly easy in the star configuration (Vicuña, 2010)

$$
\begin{gathered}
v_{\text {ringWPS2 }}=-\frac{36}{86} v_{\text {sunWPS2 }} \approx-5.23 \mathrm{~Hz}, \\
v_{\text {planetsWPS2 }}=\frac{86}{25} v_{\text {ringWPS2 }}=-18.00 \mathrm{~Hz},
\end{gathered}
$$

and the mesh frequency $v_{\text {meshWPS2 }}=36 \cdot v_{\text {sunWPS2 }}=$ $450.00 \mathrm{~Hz}$. The full revolution time calculation leads to congruence equations

$$
\begin{aligned}
& 86 \cdot N_{\text {ringWPS } 2}=0 \quad \bmod 36, \\
& 86 \cdot N_{\text {ringWPS } 2}=0 \quad \bmod 25,
\end{aligned}
$$

whose solutions are $18 \mathrm{~m}$ and $25 \mathrm{~m}$ respectively for all $m \in \mathbb{Z}$. The smallest combined solution is $\operatorname{lcm}(18,25)=$ 450 and thus 450 revolutions of the ring gear takes 450 . $1 / v_{\text {ringWPS2 }}=86.00$ seconds.

Gearbox 1 is in planetary configuration, but in contrast to the planetary gearboxes in the LHD, it is driven in the other direction (to increase the rotational speed) and the sun gear (31 teeth, output) has more teeth than the planetary gears (25 teeth). The stationary ring gear has 81 teeth. We have $v_{\text {SunWPS1 }}=v_{\text {ringWPS2 }}$ (this middle part of the two gearboxes is a floating installation) and the other frequencies are

$$
\begin{aligned}
v_{\text {carrierWPS1 }}=\frac{31}{31+81} v_{\text {sunWPS1 }} & =\frac{31}{112} v_{\text {sunWPS } 1} \\
& \approx-1.45 \mathrm{~Hz}, \\
v_{\text {planetsWPS1 }} & =-\frac{81-25}{25} v_{\text {carrierWPS1 }} \\
& =-\frac{56}{25} v_{\text {carrierWPS } 1} \approx 3.24 \mathrm{~Hz},
\end{aligned}
$$

and finally the mesh frequency

$$
v_{\text {meshWPS1 }}=81 *\left|v_{\text {carrierWPS1 }}\right| \approx 117.31 \mathrm{~Hz} .
$$

Again, to calculate the time for a full revolution we get

$$
\begin{aligned}
& 112 \cdot N_{\text {carrierWPS } 1}=0 \quad \bmod 31, \\
& 56 \cdot N_{\text {carrierWPS1 }}=0 \quad \bmod 25,
\end{aligned}
$$

whose solutions are $31 \mathrm{~m}$ and $25 \mathrm{~m}$ respectively for all $m \in$ $\mathbb{Z}$ and $\operatorname{lcm}(31,25)=775$. This takes $775 \cdot 1 / \nu_{\text {carrierWPS } 1} \approx$ 535.11 seconds.

Before the previous breakdown of gearbox 1 several years ago, it had a slightly different set of gears with 35 teeth in the sun gear, 27 in the planetary gears and 91 in the ring gear. These give the same ratio for the output of the gearbox, but the frequency of the planetary gears was $3.45 \mathrm{~Hz}$ and most importantly the mesh frequency was
$131.80 \mathrm{~Hz}$. Unfortunately, these older tooth numbers were still thought to be valid for quite some time after the breakdown probably due to some problems with communication. Obviously the lack of such mesh frequency and the the appearance of the actual mesh frequency $117.31 \mathrm{~Hz}$ in the spectra was a puzzle in the analysis of vibration measurements from this gearbox. These erroneous calculations and hardly satisfying explanations of their results ended up into some publications (Immonen et al, 2012; Nikula et al, 2015). It seems that the new gearbox is an improved design, since with these older gear tooth numbers we get full revolution of the gearbox in only 135 revolutions of the carrier (which happens due to one of the congruence equations having solutions which repeat very often), which is just 93.21 seconds, considerable less than the $535.11 \mathrm{~s}$ of the new gearbox.

\section{Calculations from the LHD mea- surements}

For calculations signals were selected from the beginning of most measurement days, when the rotational frequency of the drive shaft was approximately $13.5 \mathrm{~Hz}$. At first this was done manually but after a while with the help of an algorithm which searched for signals with constant enough tacho pulse separations to indicate the desired speed. All of these were still visually checked to select the signals for calculations. Only signals from the beginning of the workday or right after the LHD had been in its weekly maintenance were selected, because then we knew that its bucket was empty and thus the load on the axle was consistent. One could also use the tachometer signal for order tracking the signal, i.e. interpolating the signal to exact revolutions of the cardan axle. This would probably reduce variance in the calculations and make spectral analysis more exact, but because the tacho pulse was recorded badly at times, it would not have been simple to implement. From each signal a $4 \mathrm{~s}$ sample was multiplied with the window function (5) using $\varepsilon=10$ and this new signal was differintegrated with the algorithm (4). Band-pass filtering was performed with an ideal filter at cut-off frequencies $3 \mathrm{~Hz}$ and $5000 \mathrm{~Hz}$. From each end of the signal $20 \%$ was rejected and the remaining 2.4 second signal (approximately the time it takes for the carrier to rotate once) was used in the calculation of generalised $l_{p}$-norms. All the calculations were performed with Matlab.

Figure 1 shows the trend of values ${ }^{2.4} \boldsymbol{M I T}{ }_{4}^{2}$ from the point RV and a fitted regression estimate with $h=20$. In (Nissilä et al, 2014) it was already demonstrated that the relative increase of norms of snap signals were bigger than norms of acceleration signals and that the order of norm had very little effect. The fitted regression curve is almost an increasing function with smooth steps. This could mean several different faults or faults which worsen with time. 


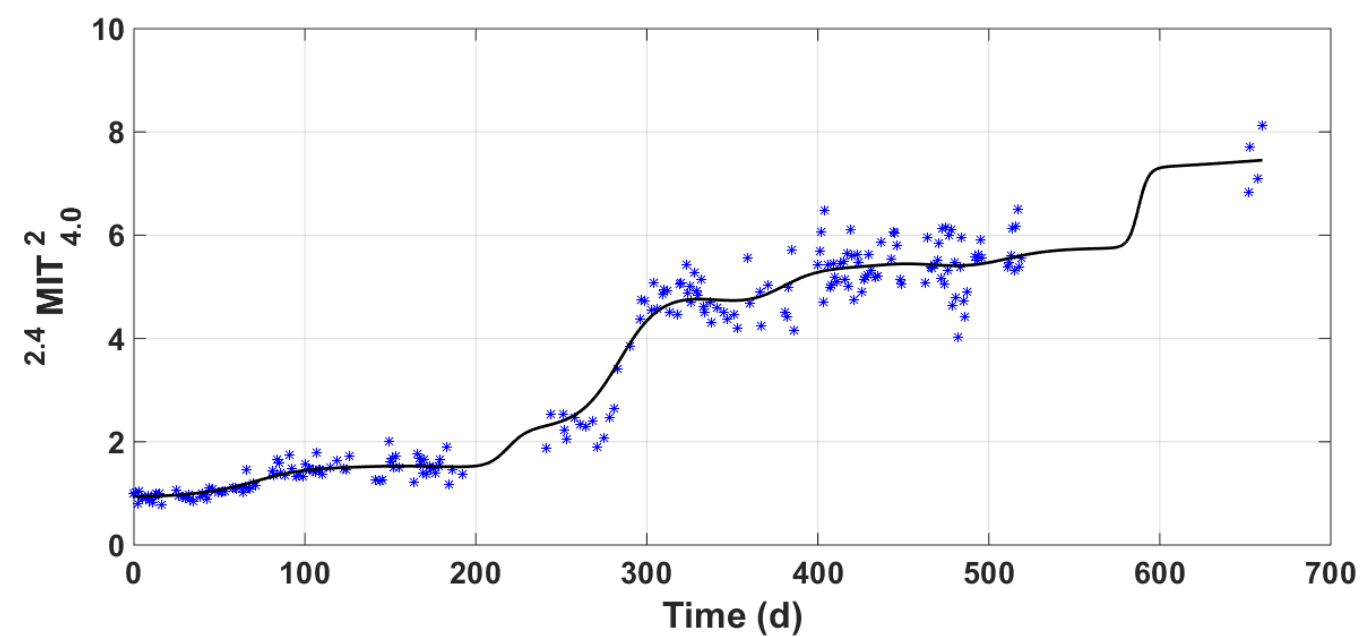

Figure 1. Trend of ${ }^{2.4} \boldsymbol{M I T}{ }_{4}^{2}$ from the point RV in the frequency range $3-5000 \mathrm{~Hz}$ and regression estimate with $h=20$

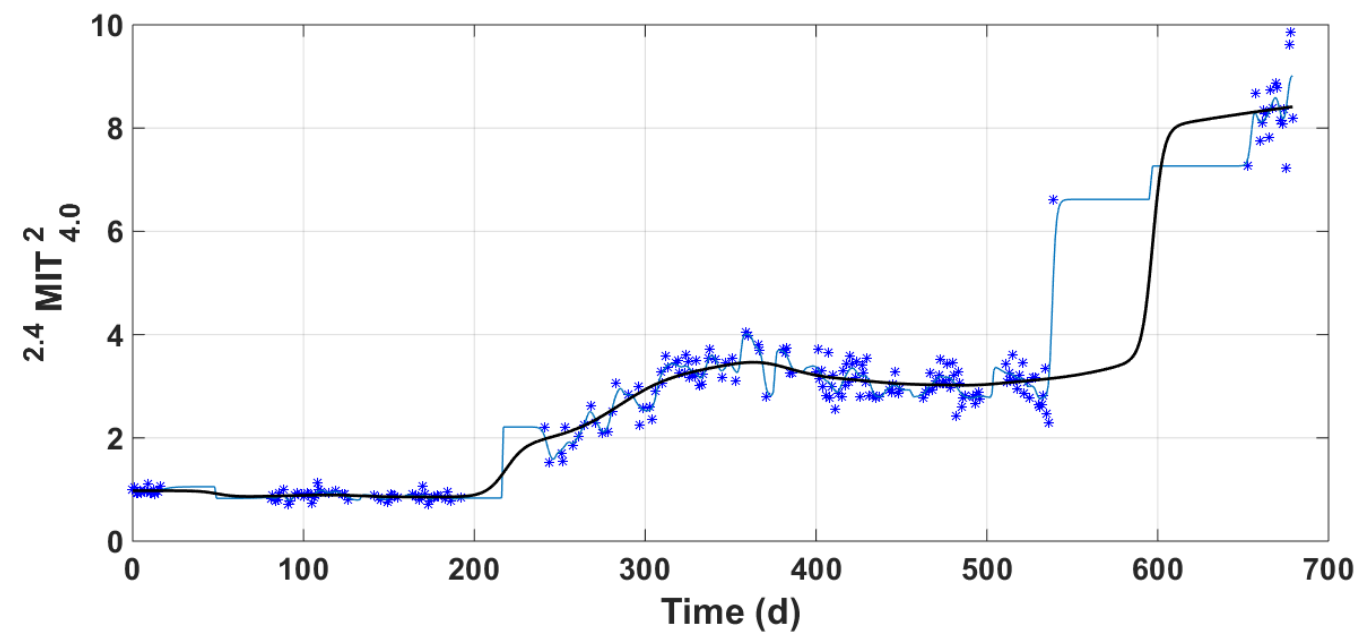

Figure 2. Trend of ${ }^{2.4} \boldsymbol{M I T}{ }_{4}^{2}$ from the point $\mathrm{LV}$ in the frequency range $3-5000 \mathrm{~Hz}$ and regression estimates with $h=20$ (black) and $h=2$ (blue)

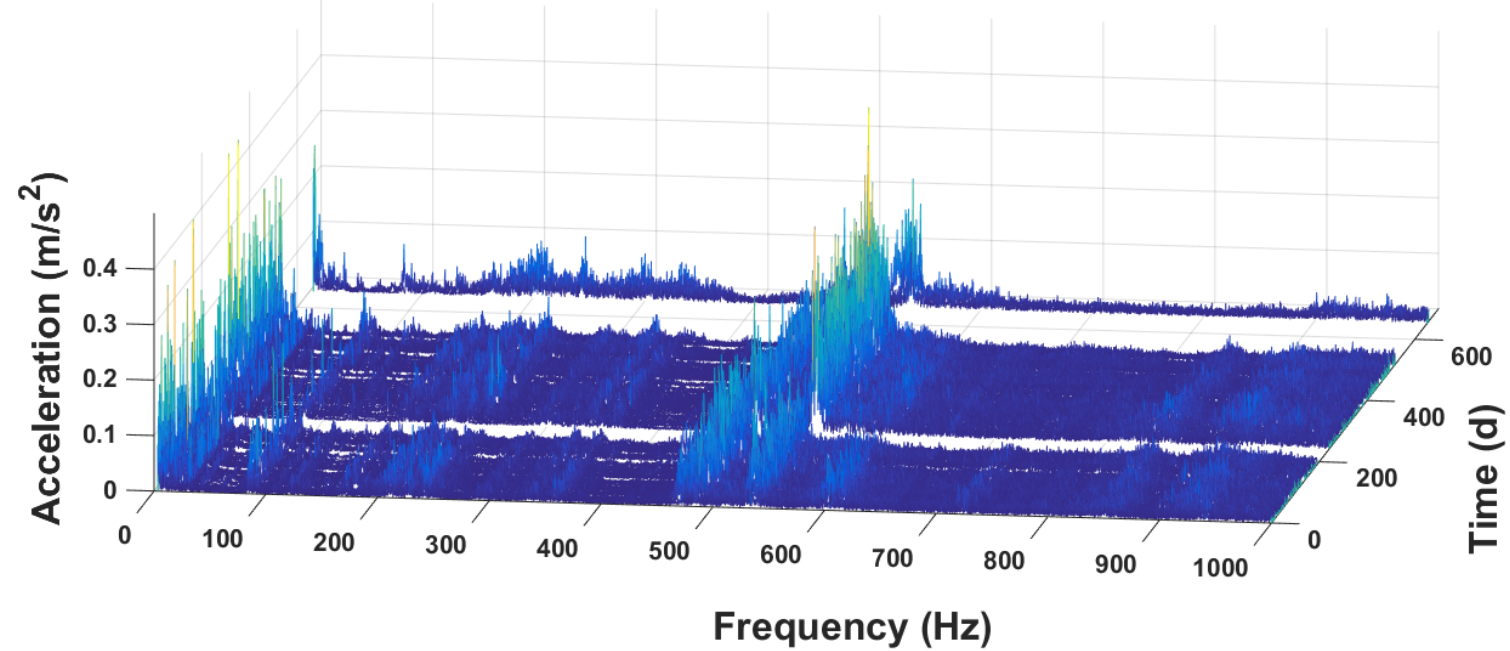

Figure 3. Waterfall plot of the spectra from the point RV in the frequency range $3-1000 \mathrm{~Hz}$ 


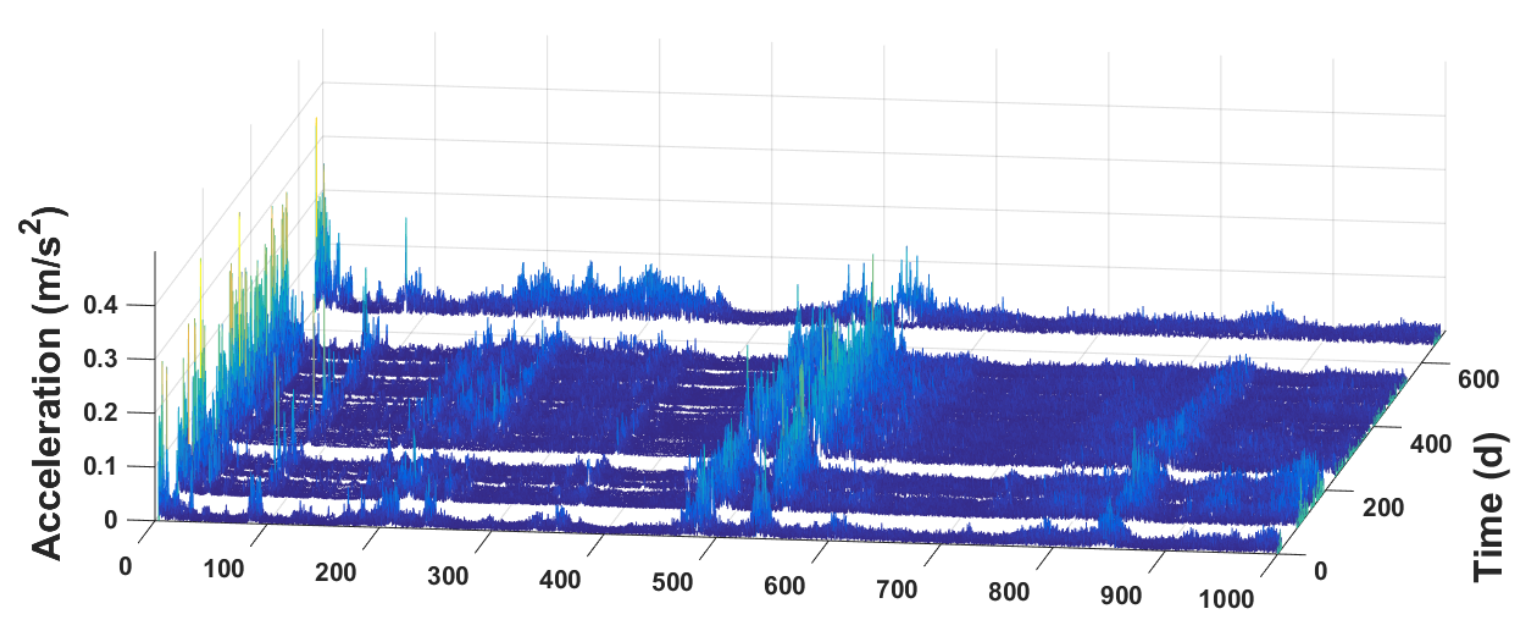

Frequency $(\mathrm{Hz})$

Figure 4. Waterfall plot of the spectra from the point $\mathrm{LV}$ in the frequency range $3-1000 \mathrm{~Hz}$

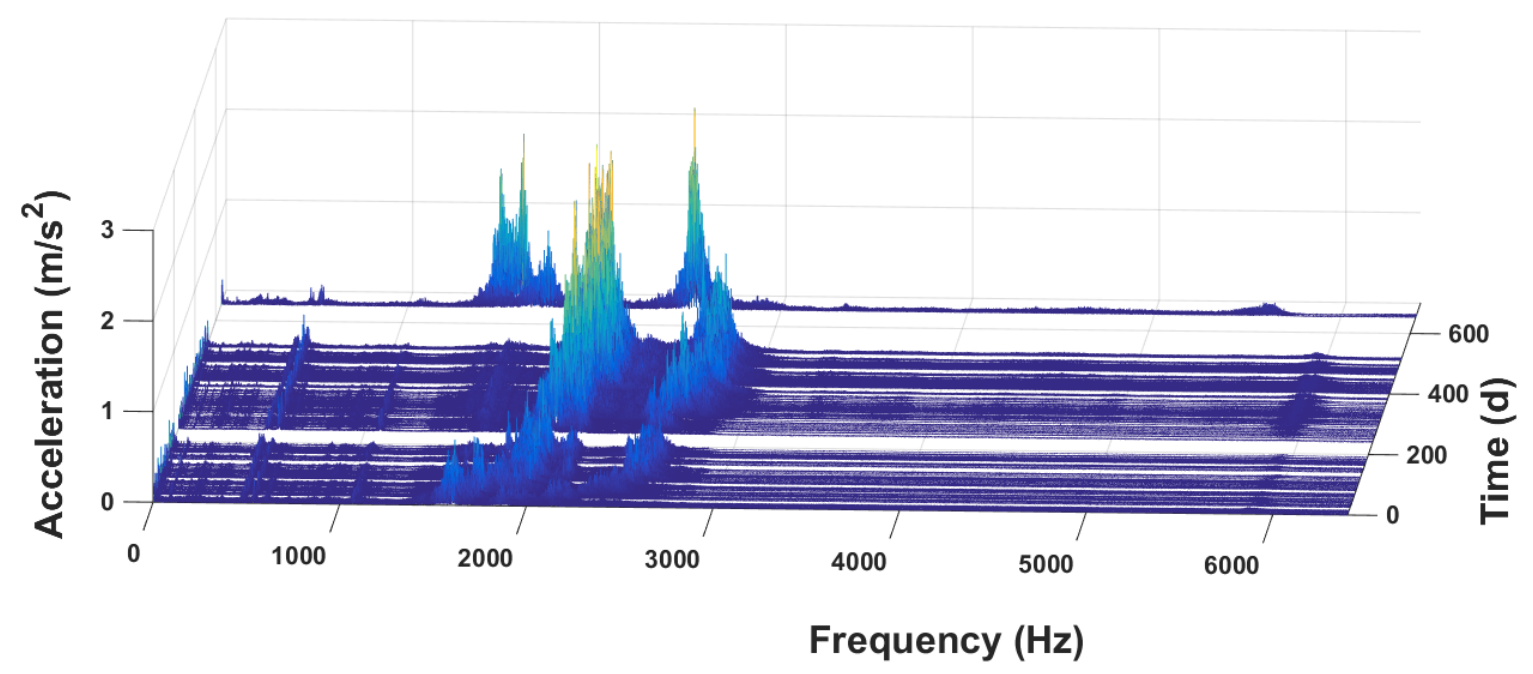

Figure 5. Waterfall plot of the spectra from the point RV in the frequency range $3-6400 \mathrm{~Hz}$

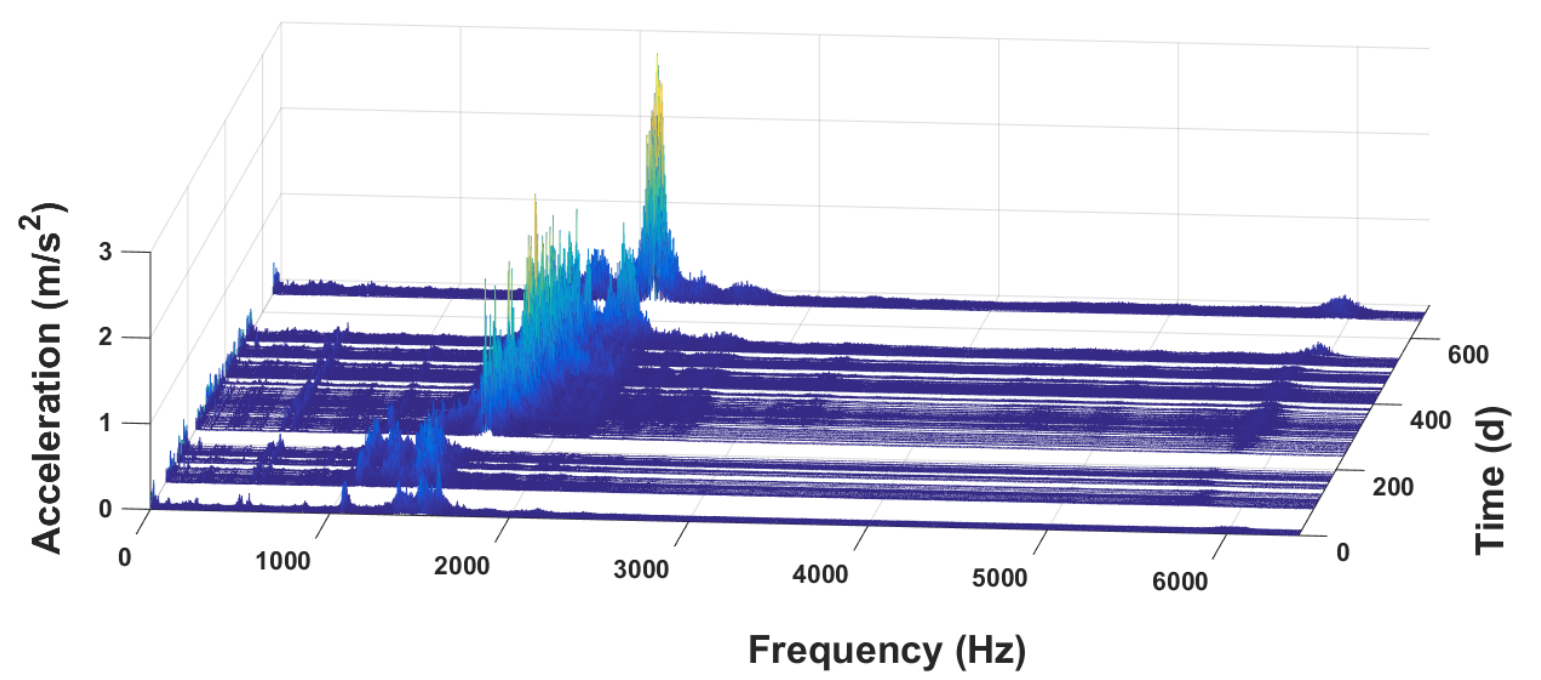

Figure 6. Waterfall plot of the spectra from the point LV in the frequency range $3-6400 \mathrm{~Hz}$ 
Figure 2 shows the trend of values ${ }^{2.4} \boldsymbol{M I T}_{4}^{2}$ from the point LV and fitted regression estimates with $h=20$ and $h=2$. After 350 days the trend starts to decrease but then makes a huge increase during the last gap in the measurements (or just before it, since there is one measurement before the gap for which ${ }^{2.4} \boldsymbol{M I T}{ }_{4}^{2}$ is almost 7). The trend estimate with $h=2$ is shown here because it manages to estimate this last big change before the gap based on that one measurement.

Fig. 3, 4, 5 and 6 show waterfall plots of the spectra which were used in the calculation of the snap signals for the trends in the previous figures. In the frequency range $3-1000 \mathrm{~Hz}$ hardly any changes occur during the measurement period. This is interesting, since this frequency range contains the mesh frequencies $(42.4 \mathrm{~Hz}$ and $121.5 \mathrm{~Hz}$ ), their multiples and other gear related vibrations. In the higher frequencies we see huge increase in vibration amplitude around $2000 \mathrm{~Hz}$ and also a drift towards higher frequencies. These spikes are probably structural resonances and one can actually see with more in depth analysis that they are mostly very high order multiples of the cardan axle frequency $13.5 \mathrm{~Hz}$. There is also increase in the $6000 \mathrm{~Hz}$ region with time. At the very end of the measurement period these high frequency resonances drop to smaller frequencies at the point RV (Fig. 5).

Some information on the damage in the axle after its breakdown was delivered to the university. It seems that the planetary gears had only minor wear on their surfaces. The ring gear of the differential had several pieces broken off from its teeth (probably explains the increase in the high multiples of the cardan frequency). There was also a lot of wear around the shafts in the axle and at least one cracked inner ring of a bearing.

\section{Calculations from the WPS mea- surements}

An overview of these measurements is provided in Fig. 7 where we have calculated the rms values from all of the vibration measurements together with the power data. It is clear that the WPS is operated very differently at different times. In the middle of summer the WPS is shut down during nights. The power output and vibration power seem to correlate so much that it is useful to investigate their relation in more detail.

Fig. 8 shows that the relation between acceleration rms and WPS power output is almost linear in gearbox 1. A good regression fit is achieved with parameter $h=0.2$. Similar linear relationships between certain vibration frequency components and WPS power output were found in a previous study from the same WPS (Nikula et al, 2015). There is a tiny flatter part around $5 \mathrm{MW}$. This flat part is more defined in the similar visualisation from gearbox 2 in Fig. 9. It seems that the higher speed gearbox 2 (which also has much bigger vibration values than gearbox 1) also exhibits more nonlinearity in WPS power vs vibration power. These figures could be used to normalise vi- bration measurements taken during different power levels of WPS in its condition monitoring. One must be careful with such methods though, since for example if the measurements from low power situations are amplified, we will also decrease the signal to noise ratio in those cases when compared to those signals which are not amplified. In what follows we will consider only measurements from the power band $7.9-8.1 \mathrm{MW}$, since then we don't need such normalisation and the four continuous measurement periods all contain measurements from this power band.

From each signal from the power band 7.9-8.1 MW we took a 6.5536 second sample (to get $6.5536 \mathrm{~s} * 5000 \mathrm{~Hz}=$ 32768 samples, a power of two) and multiplied it with the window function (5) using $\varepsilon=10$ and this new signal was differintegrated with the algorithm (4). High-pass filtering was performed with an ideal filter at cut-off frequency $3 \mathrm{~Hz}$. Because the sampling frequency was relatively low, no low-pass filtering was done. From each end of the signal $20 \%$ was rejected and the remaining 3.9 second signal was used in the calculation of generalised $l_{p}$-norms.

Fig. 10 shows the trend of values ${ }^{3.9} \boldsymbol{M I T} \boldsymbol{T}_{2}^{8}$ from gearbox 1 and a fitted regression estimate with $h=20$. We see a $10 \%$ increase in the last measurement period. Here and in the following trends we have a used a higher order norm just to show their effectiveness. In these calculations the norms with $p=8$ showed a slightly more clear increase when compared to $p=2$, but as we see in Fig. 11, the order of derivative plays a bigger role since the increase of ${ }^{3.9} \boldsymbol{M I T}_{4}^{8}$ is $20 \%$ and there is no downturn in July as there is in Fig. 10. The trend regression estimates with $h=20$ and $h=10$ only differ between the last two measurement periods as the smaller $h$ shows a more rapid increase.

Same trends are calculated from the measurements from gearbox 2 and plotted in Fig. 12 and 13. Both show a $15 \%$ increase during the measurement period, but the changes in the norm ${ }^{3.9} \boldsymbol{M I T}_{2}^{8}$ are more irregular. This is especially clear in the trend regression estimate with $h=10$ in Fig. 12. The bigger $h$ ignores these local changes and reveals the long term trend more clearly. Even so, again the snap signals are more consistent as both trend regression estimates in Fig. 13 reveal the increasing trend better than the calculations from the acceleration signals.

Waterfall plot from gearbox 1 (Fig. 14) has increased at the very end of the measurement period at 1350,1800 and $2250 \mathrm{~Hz}$, which are multiples of $v_{\text {meshWPS2 }}$. They also have sidebands $18 \mathrm{~Hz}$ apart, which is $v_{\text {planetsWPS2. The }}$ gearbox 1 mesh frequency $117.3 \mathrm{~Hz}$ is also visible and it has sidebands $1.45 \mathrm{~Hz}$ apart (not visible in this zoom level of the figure), which is $v_{\text {carrierW } P S 1}$. These do not change noticeably in the measurement period.

Waterfall plot from gearbox 2 (Fig. 15) shows hardly any change at all with time. It is a mystery why the increase at the frequencies 1800 and $2250 \mathrm{~Hz}$ are not visible here. 


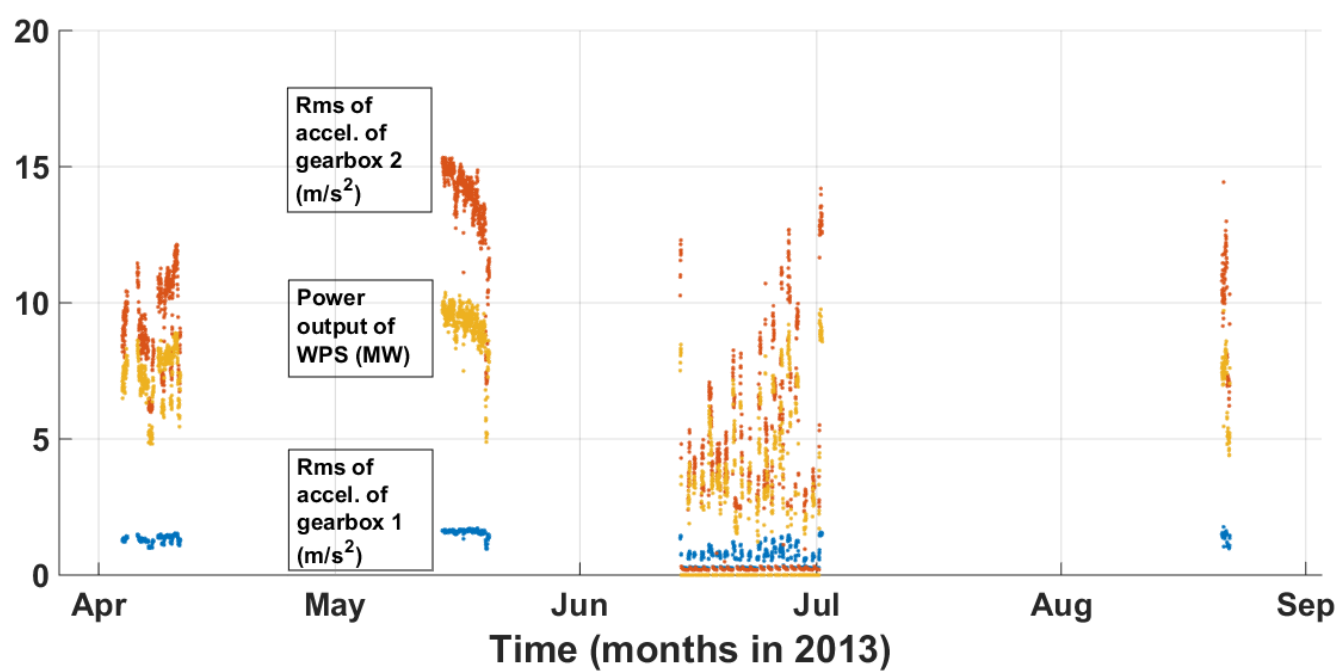

Figure 7. Overview of the measurements as rms of acceleration signals from both gearboxes and power output of WPS as functions of time

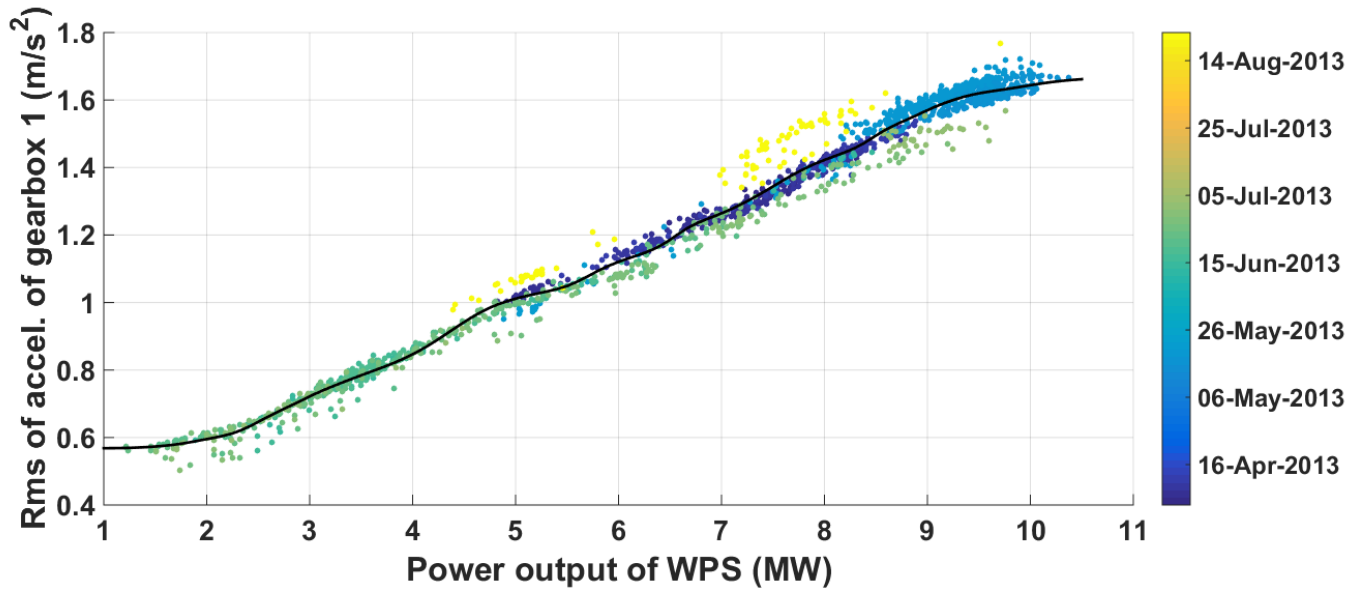

Figure 8. Power vs rms of acceleration from gearbox 1 and regression estimate with $h=0.2$, colors depict time of measurements

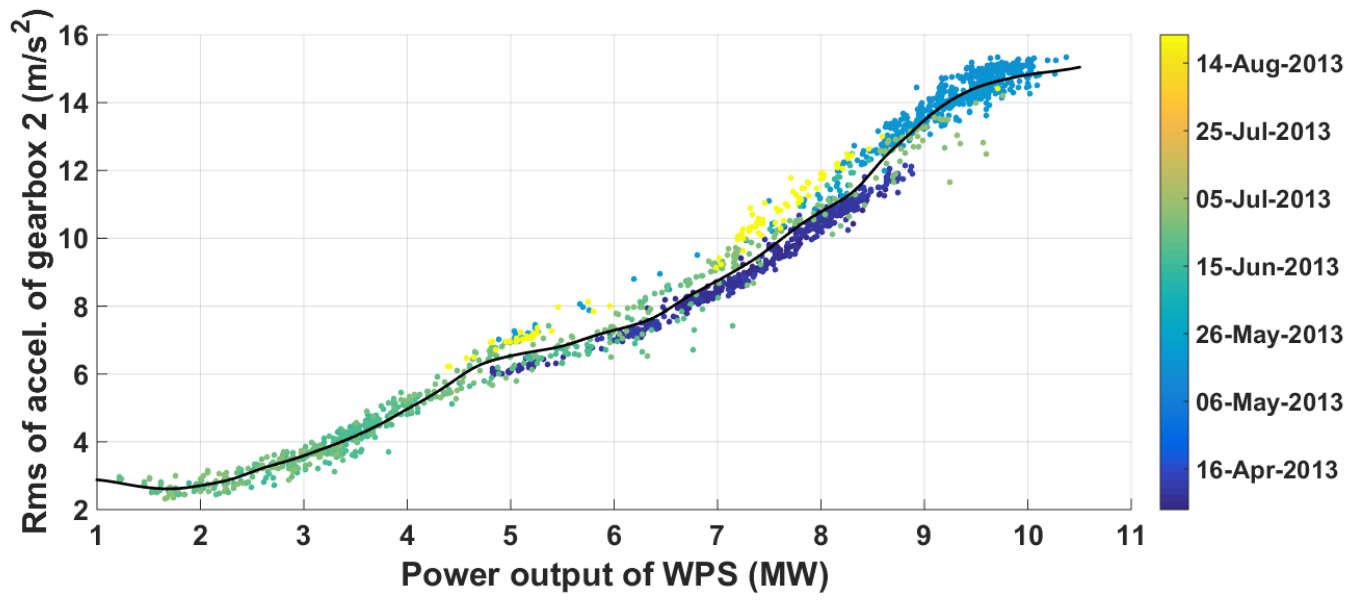

Figure 9. Power vs rms of acceleration from gearbox 2 and regression estimate with $h=0.2$, colors depict time of measurements 


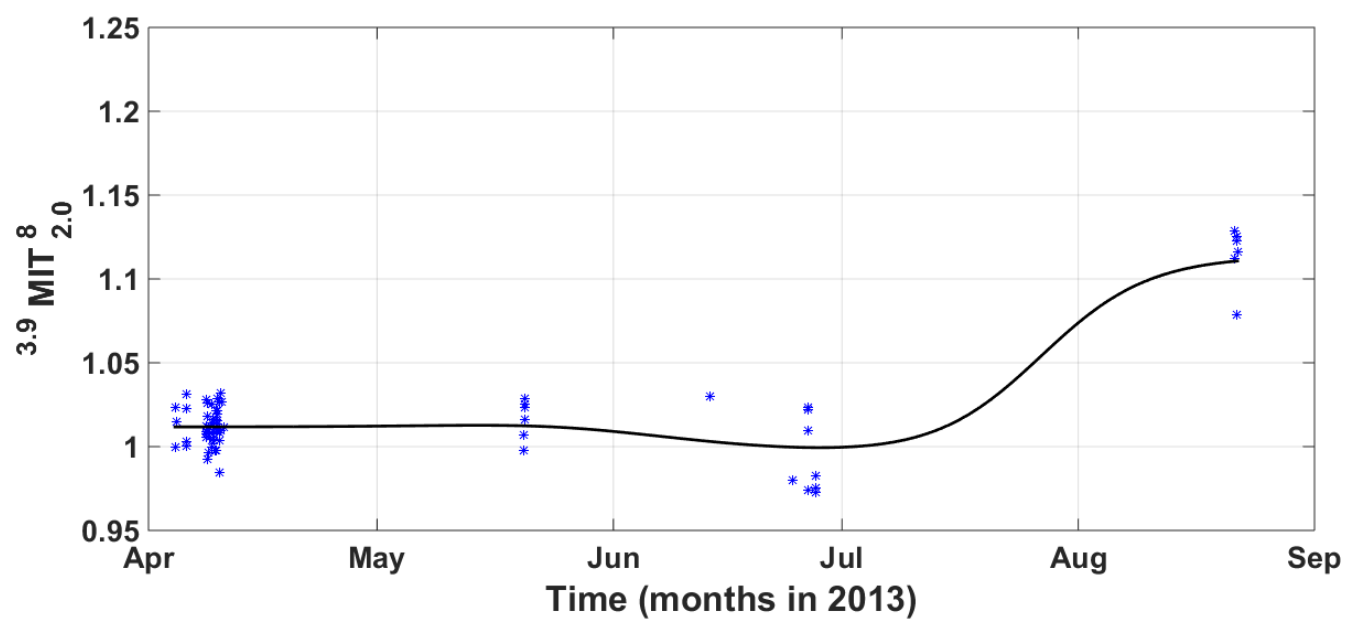

Figure 10. Trend of ${ }^{3.9} \boldsymbol{M I T}{ }_{2}^{8}$ from gearbox 1 in the frequency range $3-2500 \mathrm{~Hz}$ and regression estimate with $h=20$

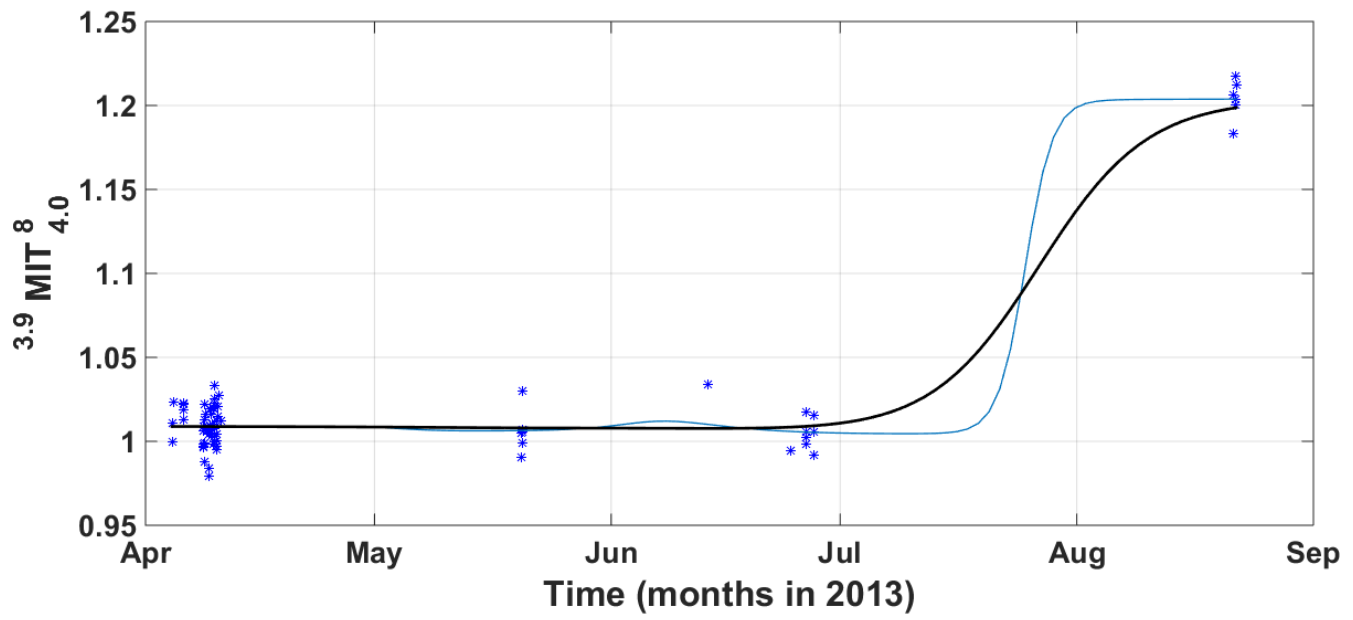

Figure 11. Trend of ${ }^{3.9} \boldsymbol{M I T}_{4}^{8}$ from gearbox 1 in the frequency range $3-2500 \mathrm{~Hz}$ and regression estimates with $h=20$ (black) and $h=10$ (blue)

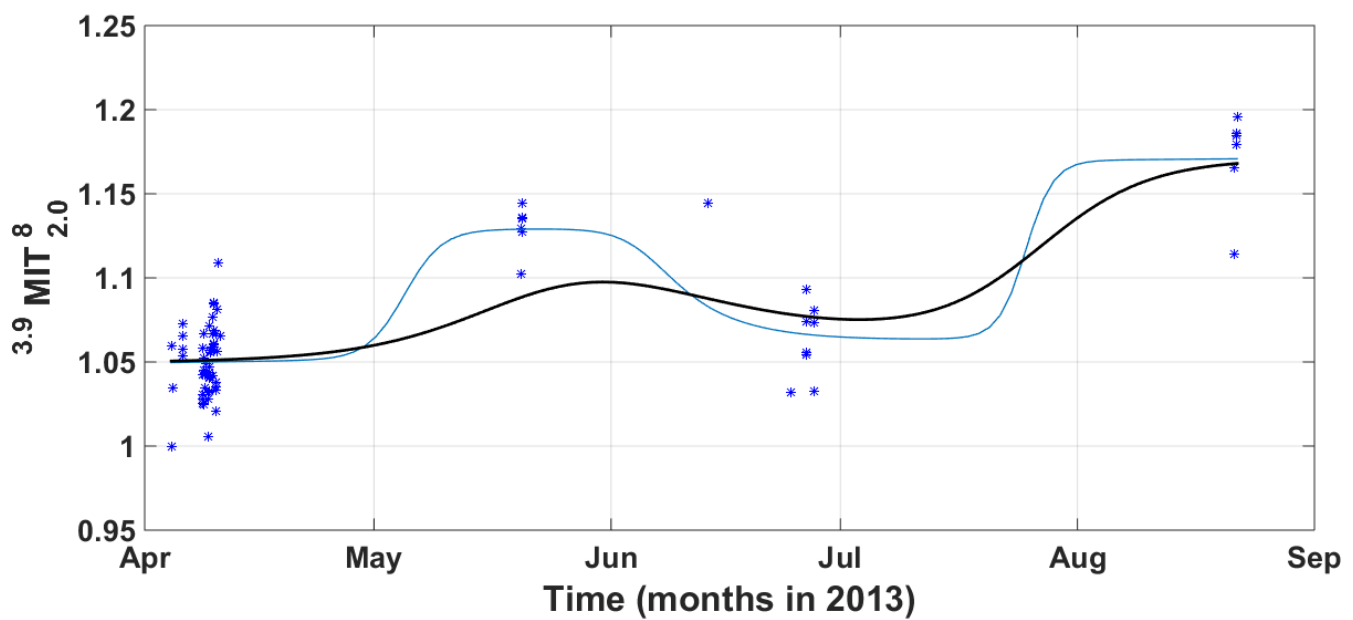

Figure 12. Trend of ${ }^{3.9} \boldsymbol{M I T}_{2}^{8}$ from gearbox 2 in the frequency range $3-2500 \mathrm{~Hz}$ and regression estimates with $h=20$ (black) and $h=10$ (blue) 


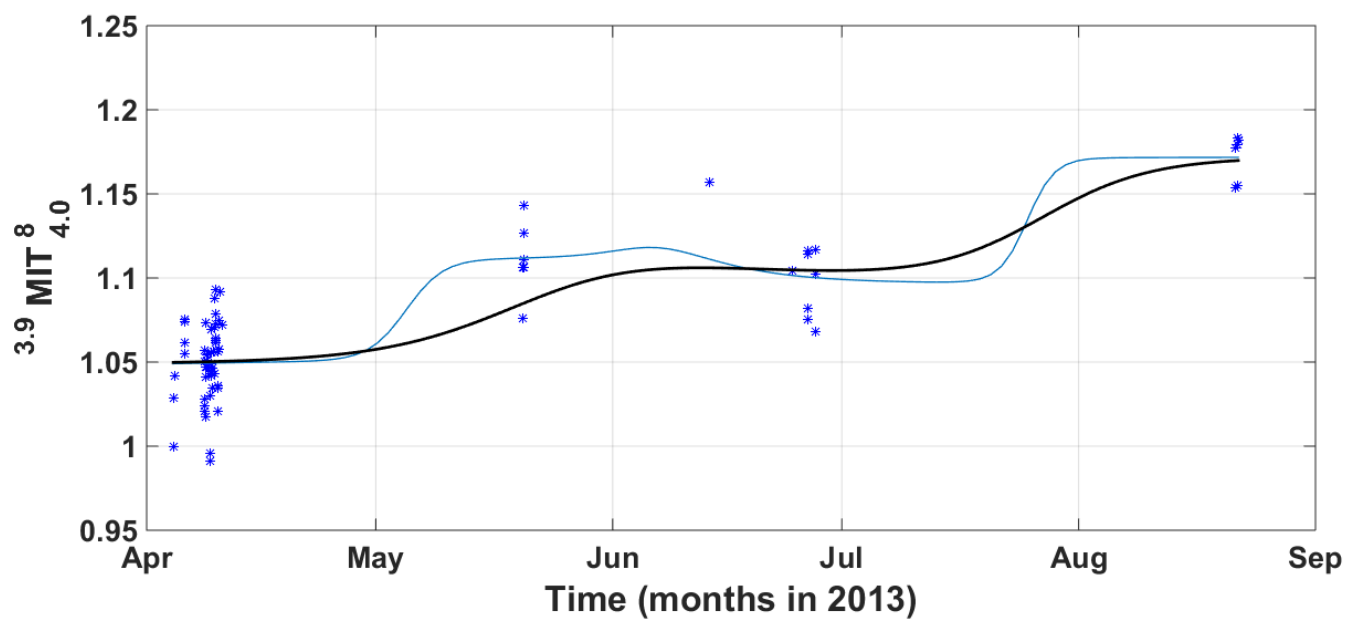

Figure 13. Trend of ${ }^{3.9} \boldsymbol{M I T}_{4}^{8}$ from gearbox 2 in the frequency range $3-2500 \mathrm{~Hz}$ and regression estimates with $h=20$ (black) and $h=10$ (blue)

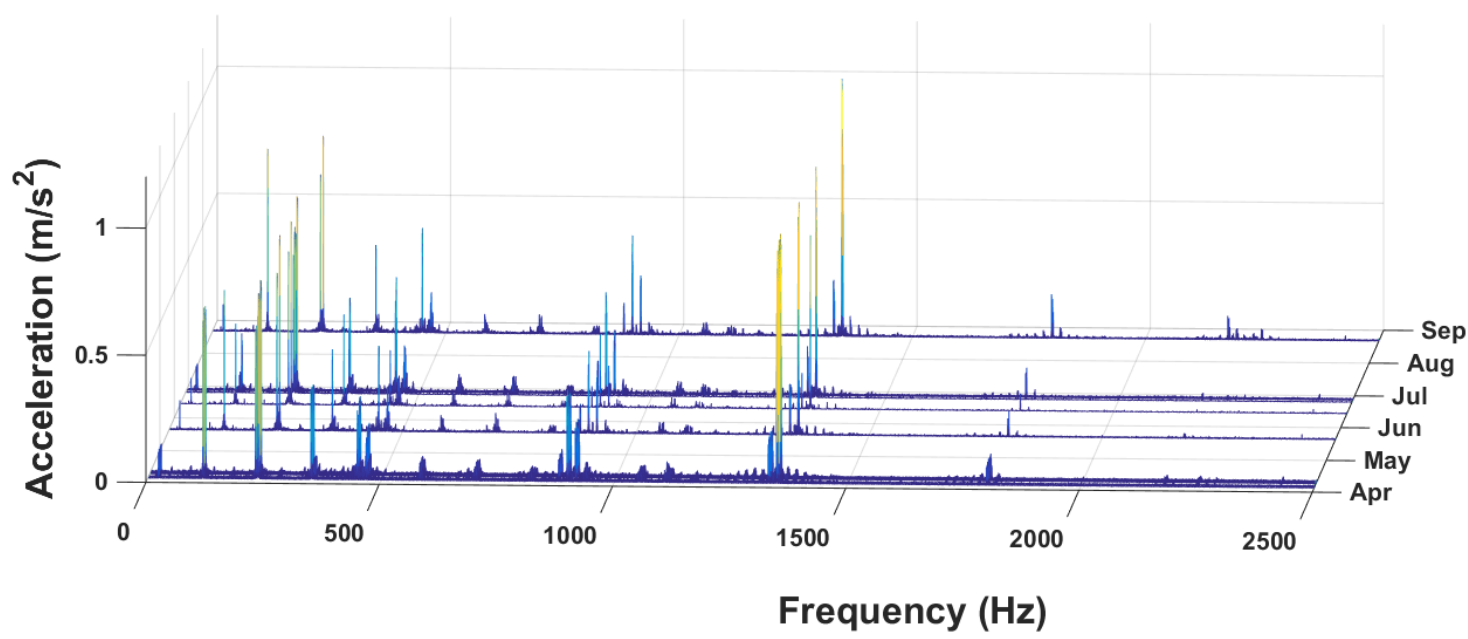

Figure 14. Waterfall plot of the spectra from gearbox 1 in the frequency range $3-2500 \mathrm{~Hz}$

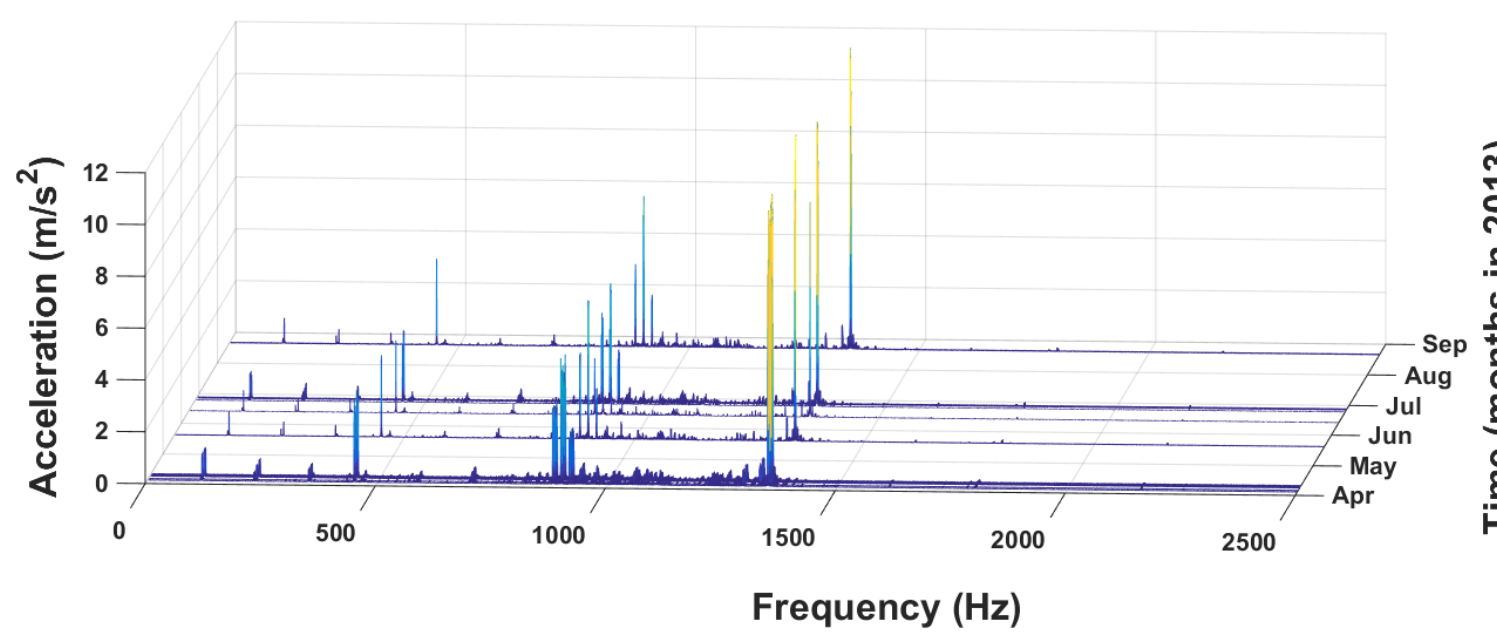

Figure 15. Waterfall plot of the spectra from gearbox 2 in the frequency range $3-2500 \mathrm{~Hz}$ 


\section{Conclusion}

The long measurement period and the final breakdown of the front axle of the LHD makes it quite clear that vibration severity calculations could serve as indicator's of its condition. Snap signals are especially sensitive for deteriorating condition of the axle. Most of the critical faults occurred in the differential gearbox, which might explain why the spectral analysis only shows noticeable increase in the multiples of the cardan frequency.

Unfortunately the data from the WPS was intermittent and lasted only for one summer period, so we can not say for certain how big are for example its typical seasonal changes in vibration severity. An increasing trend was observed from both gearboxes especially when $M I T$-indices were calculated from snap signals. An increase in the multiples of the gearbox 2 mesh frequency was also observed, but interestingly from the gearbox 1 vibration measurements.

\section{Acknowledgement}

The LHD measurements were part of the "Development of production integrated condition-based maintenance model for mining industry (DEVICO)" project. The measurements at the WPS were part of the "Integrated conditionbased control and maintenance (ICBCOM)" project. The first author wishes to thank Tauno Tönning foundation for their support on his doctoral studies.

\section{References}

W. Briggs and V.E. Henson. The DFT An Owner's Manual for the Discrete Fourier Transform. Society for Industrial and Applied Mathematics, 1995. ISBN 9780898713428.

P. S. Bullen. Handbook of Means and Their Inequalities, 2nd ed. Kluwer Academic Publishers, 2003. ISBN 9781402015229.

J. Immonen, S. Lahdelma and E. Juuso. Condition monitoring of an epicyclic gearbox at a water power station. In Proc. The 53rd Scandinavian Conference on Simulation and Modelling (SIMS2012), Reykjavik, Iceland, Oct. 2012, pp. 99-105.

S. Lahdelma. New vibration severity evaluation criteria for condition monitoring. In Finnish, University of Oulu, Finland: Research report No 85, 1992, 18 pp.

S. Lahdelma and V. Kotila. Complex Derivative - A New Signal Processing Method. Kunnossapito, 19(4):39-46, 2005 .

S. Lahdelma, and E. Juuso. Signal processing and feature extraction by using real order derivatives and generalised norms. Part 1: Methodology. The International Journal of Condition Monitoring, 1(2):46-53, 2011.
A. Laukka, J. Saari, J. Ruuska, E. Juuso and S. Lahdelma. Condition based monitoring for underground mobile machines. International Journal of Industrial and Systems Engineering, 23(1):74-79, 2016.

E. A. Nadaraya. On Estimating Regression. Theory of Probability and its Applications, 9(1):141-142, 1964.

R.-P. Nikula, K. Leiviskä, K. Karioja. Epicyclic gearbox monitoring in a hydroelectric power plant with varying load. In Proc. 11th International Conference on Condition Monitoring and Machinery Failure Prevention Technologies (CM 2015 and MFPT 2015), Oxford, UK, Jun. 2015, 12 pp.

J. Nissilä, S. Lahdelma, and J. Laurila. Condition monitoring of the front axle of a load haul dumper with real order derivatives and generalised norms. In Proc. 11th International Conference on Condition Monitoring and Machinery Failure Prevention Technologies (CM 2014 and MFPT 2014), Manchester, UK, Jun. 2014, 20 pp.

J. K. Strayer. Elementary Number Theory. Waveland Press, 1994, Sect. 2.2.

C. M. Vicuña. Contributions to the analysis of vibrations and acoustic emissions for the condition monitoring of epicyclic gearboxes. Aachener Schriften zur Rohstoffund Entsorgungstechnik des Instituts für Maschinentechnik der Rohstoffindustrie, Verlag R. Zillekens, Aachen, 2010.

G. S. Watson. Smooth regression analysis. Sankhyā: The Indian Journal of Statistics, Series A, 26(4):359-372, 1964. 